See discussions, stats, and author profiles for this publication at: https://www.researchgate.net/publication/269320347

\title{
Refractive telescope systems for future cosmic microwave background polarimetry experiments
}

Conference Paper in Proceedings of SPIE - The International Society for Optical Engineering · July 2014

DOI: $10.1117 / 12.2054634$

CITATIONS

11 authors, including:

P. C. Hargrave

Cardiff University

231 PUBLICATIONS 6,657 CITATIONS

SEE PROFILE

Marcin Lukasz Gradziel

National University of Ireland, Maynooth

94 PUBLICATIONS 434 CITATIONS

SEE PROFILE

Some of the authors of this publication are also working on these related projects:

THE PILOT balloon-borne experiment View project

Superconducting Radiation Detectors View project
Giorgio Savini

University College London

322 PUBLICATIONS 19,818 CITATIONS

SEE PROFILE

Neil Trappe

National University of Ireland, Maynooth

105 PUBLICATIONS 1,651 CITATIONS

SEE PROFILE 


\title{
Refractive telescope systems for future Cosmic Microwave Background polarimetry experiments
}

\author{
Peter Hargrave*a, Giorgio Savini ${ }^{b}$, Marcin Gradziel ${ }^{c}$, Neil Trappe ${ }^{c}$, Niall Tynan ${ }^{\mathrm{c}}$, Massimo \\ Candotti ${ }^{\mathrm{c}}$, Anthony Challinor ${ }^{\mathrm{d}}$, Stig Sørenson ${ }^{\mathrm{e}}$, Peter Ade ${ }^{\mathrm{a}}$, Rashmi Sudiwala ${ }^{\mathrm{a}}$, Maarten van der \\ Vorst $^{\mathrm{f}}$ \\ ${ }^{a}$ Cardiff University, UK; \\ bUniversity College London, UK; \\ ${ }^{c}$ National University of Ireland, Maynooth, Ireland; \\ dUniversity of Cambridge, UK; \\ 'TICRA, Copenhagen, Denmark; \\ fEuropean Space Agency, ESTEC, Nordwijk, Netherlands \\ *hargravepc@cardiff.ac.uk
}

\begin{abstract}
This paper presents the key findings of an ESA-funded programme of work to investigate refractive systems and their application to precision polarimetry experiments. We briefly summarize the derivation of requirements on the optical system for CMB polarimetry, and the design of a refractive telescope system which meets these stringent requirements. An extensive programme of experimental work was undertaken in order to better understand the optical, thermal and mechanical characteristics of the lens material, and of lenses made from this material. A repeatable and controllable antireflection coating procedure was developed and validated, and used to coat lenses used in this study. Optical measurements before and after coating have been used to validate a new module for an industry-standard antenna modelling software package.
\end{abstract}

Keywords: lens, dielectric, telescope, polarimetry, modelling, satellite, coating

\section{INTRODUCTION}

The detection of the curl-like B-mode polarization of the cosmic microwave background (CMB) induced by a stochastic background of primordial gravitational waves is well recognized as a key test of cosmic inflation in the early universe. Recent ground-based experiments have reported the detection of B-mode polarization [1, 2] and placed constraints on cosmological parameters. Future satellite instruments have been proposed [3, 4] to measure this signal down to the limit set by confusion from gravitational lensing.

All refractive antenna solutions are an attractive option for future Cosmic Microwave Background (CMB) polarimetry satellite missions. They offer the potential for excellent optical performance, with superb control of systematics over a large field of view. This requires the use of an optical system with exquisite control over polarization systematics. In this paper we present a summary and key findings of an ESA-funded study for optical configurations to address these very demanding scientific requirements. The baseline design solution comprises three dielectric lenses per telescope barrel, with a separate telescope barrel for each of the five frequency bands. All lenses will be anti-reflection coated, and are required to operate below $15 \mathrm{~K}$. There has been much uncertainty over the optical performance of dielectric lenses at low temperatures, and particularly with regard to polarization effects.

The study commenced with a thorough review and re-derivation of the science performance requirements on the optical system [5]. This was followed by review and analysis of the performance of possible optical design solutions, and a systemlevel trade-off, before selecting and fully analyzing the baseline design solution [6, 7]. The selected baseline design solution was a fully refractive telescope, with an individual telescope barrel for each of the five frequency bands. The system also allows incorporation of a rotating polarization modulator for each telescope barrel, if required. 
Such a refractive telescope system requires components at low technology readiness levels (TRL). These components were identified (coated lenses, half-wave plates), and a test plan was put in place to advance the technology for coated lenses $[8,9]$. Additionally, metal-mesh filters would be required for such a system [10]. Whilst the TRL for these filters is very high (9), having flown successfully on several satellites missions (e.g. Herschel, Planck, Spitzer, ISO, Cassini), there was concern over the extreme polarization performance of these filters that would be required for a CMB polarimetry mission. Therefore, the test programme also included an element for testing the polarization performance of the filter matrix material. At the conclusion of the testing phase of the study, the telescope baseline design was updated, and the performance re-analyzed. A technology roadmap was then produced to bring all technologies to flight level. We present the key findings from each element of this study.

\section{REVIEW OF SCIENCE REQUIREMENTS}

A thorough review and re-derivation of the science requirements was conducted as part of this study [5]. The goal of a $\mathrm{CMB}$ polarimetry mission is to measure the B-mode power from primordial gravitational waves to the limit set by confusion from gravitational lensing. For multipoles $1<200$, the latter has a white-noise spectrum $C_{l}^{B} \sim 1.7 \times 10^{-6} \mu \mathrm{K}^{2}$ corresponding to uncorrelated polarization noise with $\Delta_{P} \sim 4.5 \mu \mathrm{K}$ arcmin. The level of instrument noise in the polarization maps after foreground cleaning should therefore be better than $\Delta_{P} \sim 5 \mu \mathrm{K}$ arcmin. In practice, a noise level of around $3 \mu \mathrm{K}$ arcmin should be achievable after foreground cleaning over at least $50 \%$ of the sky (and rather more with prior information on the angular uniformity of the spectral behaviour of the foregrounds). This translates into a requirement on the number of detectors required to meet this sensitivity for a set mission duration [5]. The baseline design assumes feedhorn-coupled detectors [11], and hence the focal plane must be large enough to accommodate all detectors (two detectors per feedhorn, orthogonal polarizations).

The $B$-mode signal from primordial gravitational waves falls rapidly on scales below one degree (roughly the angle subtended by the horizon at last scattering). High angular resolution is therefore not needed for observations of the primordial signal. For example, for a resolution of 1 degree (FWHM), the signal power spectrum is suppressed by only $40 \%$ at $l=100$. Therefore we place a requirement that the beam FWHMs in the frequency range $70-353 \mathrm{GHz}$ be less than 1 degree. There is, however, a strong scientific case for improving the resolution of a future $B$-mode mission to levels of a few arcmin. Some of the key science enabled by better resolution is as follows:

- Polarization can significantly improve the lensing science that can be done with CMB surveys (e.g. Refs. [12, 13]). In particular, only with polarization can all of the information in the lensing deflection field be recovered on all scales where linear perturbation theory is reliable. This enables new science such as detailed exploration of neutrino masses with the CMB alone. Lensing science is a significant part of the science cases for COrE [3] and the intermediate [14] and comprehensive [15] EPIC mission scenarios. It calls for sensitivity of a few $\mu \mathrm{K}$ arcmin (to image lens-induced $B$-modes) and resolution of a few arcmin.

- High angular resolution $E$-mode polarization observations can improve constraints on primordial non-Gaussianity (for example, by around a factor of two in local models [16]). Non-Gaussian science is identified as an important part of the science case for COrE. The requirements are similar to those for lensing.

Other ancillary science such as Galactic magnetic fields and their role in star formation, the properties of interstellar dust and the discovery and characterization of polarized extra-galactic sources requires arcmin angular resolution and broad frequency coverage. Improved resolution can also indirectly benefit the recovery of large-angle $B$-modes in the following ways.

- The deflection field reconstructed from high-resolution CMB polarization observations can be used to "delens" approximately the CMB thus reducing the impact of lensing noise on determinations of the $B$-mode power from gravitational waves [13].

- Many main-beam systematic effects, such as beam ellipticity and differential pointing (of a given fraction of the beam width) are suppressed below the beam scale. The spurious $B$-mode power they generate is proportional to powers of $l \sigma$ (where $\sigma=\mathrm{FWHM} / \sqrt{8 \ln 2}$ ). Improved resolution lessens the impact of such beam systematics on the large scales relevant for primordial $B$-mode searches.

Accepting that high-l CMB science and Galactic science are not the main drivers for the mission considered here, we specify goal beam sizes such that the finite resolution has a negligible impact on the power spectrum sensitivity at $l=100$. 
This is achieved with 20 -arcmin resolution at $100 \mathrm{GHz}$, which should also result in only a small loss of survey area due to point-source masking.

Generally, polarization purity is more important than higher resolution for a large-angle B-mode mission and should not be compromised in favour of meeting the goal resolution.

We selected five bands which would allow us to achieve the mission sensitivity goals of $3 \mu \mathrm{k}$ in $30 \%$ spectral bands at 70 , $100,143,217 \mathrm{GHz}$, and $10 \mu \mathrm{K}$ at $353 \mathrm{GHz}$. The choice of frequency coverage and the sensitivity at each frequency is dictated by the need to remove diffuse foregrounds aggressively on large scales. Here, we did not attempt to provide a detailed optimization of the frequency coverage in light of expected foregrounds. Rather, we test whether the frequency coverage of a suggested baseline mission, and its goal and required sensitivities, allow cleaning of foregrounds to levels that would not compromise the mission goals. The diffuse foregrounds that we expect to be relevant in polarization are synchrotron emission and thermal dust emission from partially-aligned aspherical grains. Details of the analyses can be found in [5].

Table 1. Frequency bands, beam sizes (full-width at half-maximum; FWHM) and goal and required polarization sensitivities of the baseline mission

\begin{tabular}{|c|c|c|c|}
\hline$v(G H z)$ & FWHM (arcmin) & $\begin{array}{c}\boldsymbol{\Delta}_{\mathbf{p}} \text { (goal) } \\
(\boldsymbol{\mu K} \text { arcmin) }\end{array}$ & $\begin{array}{c}\boldsymbol{\Delta}_{\mathbf{p}} \text { (requirement) } \\
(\boldsymbol{\mu} \mathbf{K} \text { arcmin) }\end{array}$ \\
\hline 70 & 30 & 3 & 10 \\
\hline 100 & 20 & 3 & 5 \\
\hline 143 & 15 & 3 & 5 \\
\hline 217 & 10 & 3 & 10 \\
\hline 353 & 10 & 10 & \\
\hline
\end{tabular}

We find that using the five frequencies of the baseline mission scenario (Table 1), it is possible to remove foregrounds to the levels required to achieve the mission's goals. For the goal and required sensitivities in Table 1, effective noise levels in the cleaned $\mathrm{CMB}$ should meet the mission goals and requirements over significant ( $>50 \%)$ fractions of the sky. Using prior information on the spectral behaviour of the foregrounds is important and should improve the CMB reconstruction in regions of high emission. Finally, modelling the dust emission beyond a power-law will be required to eliminate foreground residuals at the $\sim 0.3 \mu \mathrm{K}$ level.

Exquisite control of systematic effects is crucial for B-mode polarimetry, and we require excellent knowledge of the systematic effects induced by the optical system itself. It is very important to minimize instrumental polarization (leakage from temperature signal into the much weaker polarization). We set a specification on integrated cross-polar levels better than $-30 \mathrm{~dB}[5,17]$.

A necessary requirement of any future B-mode mission is that it can accurately characterize the $B$-mode spectrum from primordial gravitational waves if the tensor-to-scalar ratio $r>10^{-2}$. The BICEP2 team have recently reported a measurement of $r=0.20_{-0.05}^{+0.07}$ [1]. At this level, a detection should certainly be achievable from sub-orbital experiments. The job of the space mission would then be to provide a definitive measurement of $r$, characterize the B-mode spectrum of gravitational waves and demonstrate robustness of the results to the treatment of foregrounds and systematic effects. A natural requirement to demonstrate robustness is to have the instrument noise comparable to the lensing "noise" in all the CMB frequency channels. Furthermore, the mission should have sufficient frequency coverage and sensitivity in its foreground-dominated channels to achieve an effective noise level after foreground cleaning comparable to the lensing noise over a significant fraction of the sky. The required sensitivities for the baseline mission in Table 1 meet this first requirement, and also meets the second requirement over more than $50 \%$ of the sky.

The baseline scan strategy considered is similar to that proposed for B-Pol and EPIC [14]. The payload rotates about its spin axis with a period of $\tau_{s}$ and the centre of the focal plane is offset from the spin axis by the boresight angle $\theta_{b}$. The spin axis precesses on a cone with semi-angle $\theta_{p}$ with period $\tau_{p}$. The axis of the cone rotates with a period of one year to remain always anti-solar. The resulting scan pattern provides good angular coverage in any pixel and provides wellsampled maps of more than half the sky per day (once averaged over the focal plane). The effective sensitivity is a function of mission duration. We place a recommendation that the mission duration be of order 4 years, based on funding and 
satellite resource limitations. This mission duration allows the sensitivity requirements to be met with a realistic number of detectors. The detector numbers are calculated based on the assumption of the use of background-limited detectors, and that the total optical loading is a factor of 1.5 times that from the CMB alone. We assumed the use of feedhorn-coupled detectors for this study, with two-detectors per horn (one per polarization). The intrinsic cross-polarization of the feed puts a limit on the performance of the optical system. In the case of ultra-Gaussian horns [11], which were used for the analyses presented here, the cross-polar power level is very low. Additionally, we required excellent knowledge of the antenna feed characteristics, and we only had this information for the ultra-Gaussian feedhorns.

The derivation of main beam and sidelobe requirements were used, together with the derivation of detector requirements, to produce a summary of requirements on the optical system, presented in Table 2 . The requirements are derived assuming that the beam is a good fit to an elliptical Gaussian distribution on the celestial sphere, the fit being made to the power pattern over the main beam.

Table 2. Requirements on optical system $(\mathrm{R}=$ requirement, $\mathrm{G}=\mathrm{goal})$. $\uparrow$ Note that these numbers are stated assuming a 4-year mission duration. \$Quadrupole and dipole co-polar beam power requirements assume goal angular resolution

\begin{tabular}{|c|c|c|c|c|c|c|}
\hline \multicolumn{2}{|l|}{ Frequency $(\mathrm{GHz})$} & 70 & 100 & 143 & 217 & 353 \\
\hline \multicolumn{2}{|l|}{ Bandwidth } & $30 \%$ & $30 \%$ & $30 \%$ & $30 \%$ & $30 \%$ \\
\hline \multicolumn{2}{|l|}{ Optical efficiency } & $>60 \%$ & $>60 \%$ & $>60 \%$ & $>60 \%$ & $>60 \%$ \\
\hline \multicolumn{2}{|l|}{$\begin{array}{l}\text { Out of band rejection } \\
(>1 \mathrm{THz})\end{array}$} & $>120 \mathrm{~dB}$ & $>120 \mathrm{~dB}$ & $>120 \mathrm{~dB}$ & $>120 \mathrm{~dB}$ & $>120 \mathrm{~dB}$ \\
\hline \multirow{2}{*}{$\begin{array}{l}\text { Resolution } \\
\text { (arcmin) }\end{array}$} & $\mathbf{G}$ & 30 & 20 & 15 & 10 & 10 \\
\hline & $\mathbf{R}$ & $<50$ & $<50$ & $<50$ & $<50$ & $<50$ \\
\hline \multirow{2}{*}{$\begin{array}{l}\text { Sensitivity } \\
(\mu K . \operatorname{arcmin})\end{array}$} & $\mathbf{G}$ & 3 & 3 & 3 & 3 & 10 \\
\hline & $\mathbf{R}$ & 10 & 5 & 5 & 5 & 10 \\
\hline \multirow[t]{2}{*}{ \# Detectors $\dagger$} & G & 836 & 776 & 776 & 984 & 240 \\
\hline & $\mathbf{R}$ & 76 & 280 & 280 & 354 & 240 \\
\hline \multirow{2}{*}{$\begin{array}{l}\text { \# Pixels on focal } \\
\text { plane } \dagger\end{array}$} & $\mathbf{G}$ & 418 & 388 & 388 & 492 & 120 \\
\hline & $\mathbf{R}$ & 38 & 140 & 140 & 177 & 120 \\
\hline \multirow{2}{*}{$\begin{array}{l}\text { Beam ellipticity (@) } \\
-3 \mathrm{db})\end{array}$} & G & 0 & 0 & 0 & 0 & 0 \\
\hline & $\mathbf{R}$ & $<07$ & $<0.7$ & $<0.7$ & $<0.7$ & $<0.7$ \\
\hline \multirow{2}{*}{$\begin{array}{l}\text { Quadrupole co- } \\
\text { polar beam power } \\
(\mathrm{dB}) \uparrow\end{array}$} & $\mathbf{G}$ & -22.9 & -16.2 & -11.3 & -4.4 & 6.1 \\
\hline & $\mathbf{R}$ & -19.0 & -11.9 & -6.9 & 0.1 & \\
\hline \multirow{2}{*}{$\begin{array}{l}\text { Dipole co-polar } \\
\text { beam power }(\mathrm{dB}) \ddagger\end{array}$} & $\mathbf{G}$ & -28.3 & -25.1 & -22.7 & -19.3 & -8.8 \\
\hline & $\mathbf{R}$ & -24.3 & -20.8 & -18.3 & -14.8 & \\
\hline \multirow[t]{2}{*}{ Directivity } & G & 0.99 & 0.99 & 0.99 & 0.99 & 0.99 \\
\hline & $\mathbf{R}$ & 0.95 & 0.95 & 0.95 & 0.95 & 0.95 \\
\hline \multirow{2}{*}{$\begin{array}{l}\text { Sidelobe power } \\
\text { ratio }(\mathrm{dB})\end{array}$} & G & -54.5 & -55.7 & -58.6 & -63.6 & -67.3 \\
\hline & $\mathbf{R}$ & -45.8 & -47.4 & -50.5 & -55.5 & \\
\hline \multirow{2}{*}{$\begin{array}{l}\text { Beam mismatch } \\
(\mu) \ddagger\end{array}$} & $\mathbf{G}$ & $1.8 \times 10^{-3}$ & $3.9 \times 10^{-3}$ & $6.8 \times 10^{-3}$ & $1.5 \times 10^{-2}$ & $5.1 \times 10^{-2}$ \\
\hline & $\mathbf{R}$ & $2.8 \times 10^{-3}$ & $6.3 \times 10^{-3}$ & $1.1 \times 10^{-2}$ & $2.5 \times 10^{-2}$ & \\
\hline \multirow[t]{2}{*}{$\Delta$ Ellipticity $(\Delta \varepsilon)$} & G & $2.4 \times 10^{-3}$ & $5.2 \times 10^{-3}$ & $9.2 \times 10^{-3}$ & $2.1 \times 10^{-2}$ & $6.8 \times 10^{-2}$ \\
\hline & $\mathbf{R}$ & $3.8 \times 10^{-3}$ & $8.6 \times 10^{-3}$ & $1.5 \times 10^{-2}$ & $3.4 \times 10^{-2}$ & \\
\hline \multirow[t]{2}{*}{$\Delta$ Pointing $\left(\chi_{d}\right)$} & G & $6.2 \times 10^{-4}$ & $8.9 \times 10^{-4}$ & $1.2 \times 10^{-3}$ & $1.7 \times 10^{-3}$ & $5.8 \times 10^{-3}$ \\
\hline & $\mathbf{R}$ & $9.7 \times 10^{-4}$ & $1.5 \times 10^{-3}$ & $1.9 \times 10^{-3}$ & $2.9 \times 10^{-3}$ & \\
\hline \multirow{2}{*}{$\Delta$ Directivity $\left(\Delta_{D}\right)$} & $\mathbf{G}$ & $5.7 \times 10^{-5}$ & $5.7 \times 10^{-5}$ & $5.7 \times 10^{-5}$ & $5.7 \times 10^{-5}$ & $1.9 \times 10^{-4}$ \\
\hline & $\mathbf{R}$ & $3.3 \times 10^{-5}$ & $3.3 \times 10^{-4}$ & $3.3 \times 10^{-4}$ & $3.3 \times 10^{-4}$ & \\
\hline \multirow{2}{*}{$\begin{array}{l}\text { Common rotation } \\
(\vartheta)\end{array}$} & G & $2.3^{\prime}$ & $2.3^{\prime}$ & $2.3^{\prime}$ & $2.3^{\prime}$ & $7.6^{\prime}$ \\
\hline & $\mathbf{R}$ & $6.7^{\prime}$ & $6.7^{\prime}$ & $6.7^{\prime}$ & $6.7^{\prime}$ & \\
\hline \multirow{2}{*}{$\begin{array}{l}\text { Common pointing } \\
(\sigma \chi)\end{array}$} & G & $0.98^{\prime}$ & $0.98^{\prime}$ & $0.98^{\prime}$ & $0.98^{\prime}$ & \\
\hline & $\mathbf{R}$ & $1.5^{\prime}$ & $1.5^{\prime}$ & $1.5^{\prime}$ & $1.5^{\prime}$ & \\
\hline
\end{tabular}




\section{TELESCOPE CONFIGURATION OPTIONS}

We considered several telescope and instrument configurations, including fully reflective, fully refractive and hybrid design solutions. Hybrid solutions were discounted because of the physical size of any resulting viable system incorporating the necessarily large focal plane that would result from the use of feedhorns. Note that we only considered the use of feedhorns for this study, as we had high confidence in the modelled beams. The priority for this study was to evaluate telescope options in terms of main beam effects. This does not mean that other feed options are excluded, simply that we needed to minimize uncertainty in the feed for this study. We analysed in detail four different instrument configurations:

- Option A - Single compact range antenna with common focal plane for all frequencies,

- Option B - Multiple compact range antennae, with individual focal planes separated by frequency band,

- Option $\mathrm{C}$ - Multiple refractive telescopes, with individual focal planes separated by frequency band, sized for optimal Gaussian beam profile (GBP),

- Option C2 - Multiple refractive telescopes, with individual focal planes separated by frequency band, sized for optimal aperture efficiency (AE).

The system aperture could be designed for optimal Gaussian beam profile (GBP), or optimal aperture efficiency. Reducing the aperture will improve aperture efficiency (AE) and reduce system volume and mass, but at the expense of increasing the side-lobe level.

A detailed analysis of the optical performance of each system was then carried out, together with simulations of the effect of the optical performance on CMB parameter retrieval (science performance). Details may be found in [7]. A comprehensive trade-off analysis was then carried out for the candidate options, in the context of a future satellite implementation, where the top-level trade-off parameters were performance vs. cost vs. risk. Although the $\mathrm{C} 2$ option has significantly better scientific performance, it is only marginally superior to option B once all trade-off parameters are considered.

\section{TELESCOPE BASELINE DESIGN \& ANALYSIS}

We concluded that the optimal configuration for a space-borne CMB polarimetry experiment would be a receiver with five bands, centred at 70,100,150, 217 and $353 \mathrm{GHz}$, with an individual antenna for each channel. An individual antenna for each frequency channel allows sufficient space for the accommodation of detectors on the focal plane, and enables the optimization of anti-reflection coatings and half-wave plates (if required) for each channel. We propose two distinct telescope designs. One design for the three lowest frequency bands $(70,100,143 \mathrm{GHz})$ and another for the two higher frequency bands (217 and $353 \mathrm{GHz}$ ). The only difference between these two designs is the system aperture, and hence element diameters. This leads to two f-numbers, $\mathrm{F}_{\# 1}=2.2359$ and $\mathrm{F}_{\# 2}=3.4075$ respectively for the first three bands and the last two bands of the instrument. In all other respects, the telescopes are identical, and have the form illustrated in Figure 1.

The semi-field of view spans $9.5^{\circ}$ from the telescope axis, which at the focal surface translates into an available circular area of $164.4 \mathrm{~mm}$ radius for placement of horns. The focal surfaces were modeled according to the external diameters of the assumed ultra-Gaussian horns, allowing a packing density of $75 \%$. Horns are placed on the focal plane in concentric hexagonal rings. With this scheme, we meet the requirements on horn numbers for all bands using one telescope per frequency band.

The performance was analyzed for two cases. The first case assumed only single reflection on each lens surface, and the second case modelled the performance for the presence of multiple reflections (ghosting analysis) as if no coating layer were applied. Full details of the analyses and performance predictions may be found in [6]. The analyses were conducted using physical optics modelling with the GRASP-QUAST module (Figure 2). A detailed comparison of GRASP and CodeV modelling predictions was also performed as part of this work.

Optically induced beam ellipticity was predicted to be negligible on axis reaching $3.4 \%$ and $4.1 \%$ for orthogonal sources at the edge of the field. Beam FWHM is predicted to be 25.0 arc minutes at the centre of the field and 25.1 arc minutes at the margin. The predicted ratio of power in the main beam to power in the sidelobes over a \pm 2 degree field is greater than $98.7 \%$ over the entire field. The ratio of cross-polar to co-polar power ranges from $-57.23 \mathrm{~dB}$ on axis to $-42.62 \mathrm{~dB}$ at $9.5^{\circ}$ off axis. Main beam differential effects with and without multiple reflections were also modeled using GRASP-QUAST. 


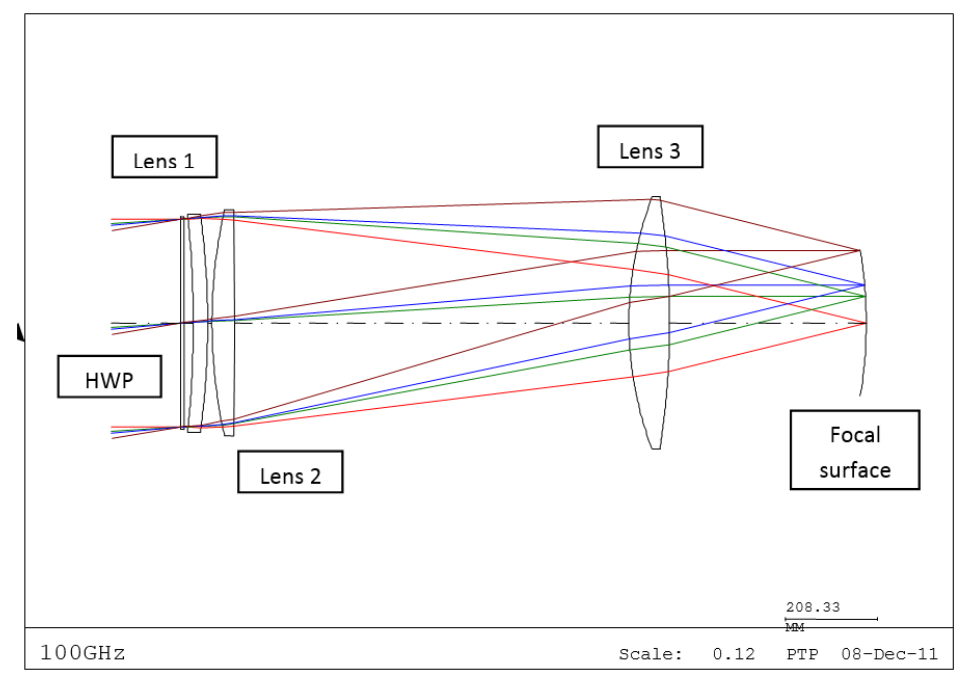

Figure 1. Baseline optical design.

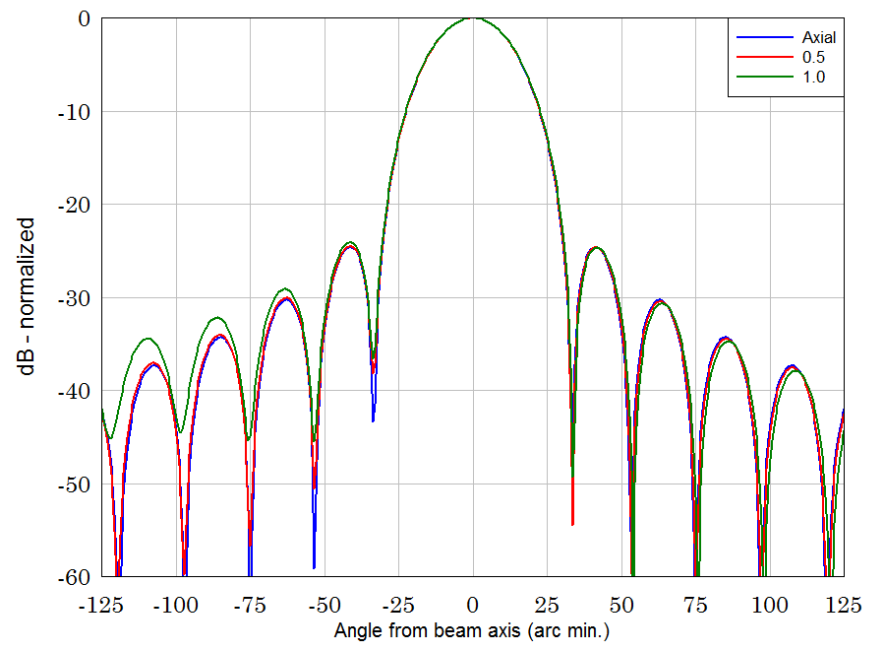

Figure 2. Far field beams from the GRASP9 PO model for the axial, mid-radial and marginal pixel in the baseline design $100 \mathrm{GHz}$ system. Note that, the marginal pixel shows marked asymmetry in the marginal beam sidelobes.

\section{Ignoring multiple reflections}

The first order differential effects analysis shows that the system performs excellently for all horn locations and polarization rotations in the focal surface, well within the performance requirements. The ellipticity of the beams is contained to within $3 \%$ for the most aberrated beams at the marginal positions.

\section{Considering multiple reflections}

The presence of multiple reflections between lenses only slightly worsens the performance of the optical system. The ellipticity of the far-field beams is $\sim 3 \%$ worse compared than for the case of no multiple reflections. There is an indication that the common pointing also degrades as horns in the focal plane move radially towards the margin of the focal surface, but it is believed that this is a fitting artifact due to the deterioration in the aberration of the beam patterns.

\section{Effects of internal reflections on beams - “double PO analysis"}

In the "Double PO" (physical optics) procedure, the internal reflections in the system includes both reflections inside the lens (between the two faces of the lens) and between lenses. The effects can be separated as shown in Figure 3 for Band 2 at $100 \mathrm{GHz}$ with on-axis feed. Here the standard PO, without internal reflections, is compared to an analysis including only internal lens reflections and to an analysis including only reflections between lenses. A final analysis including both types of reflections is also shown. As a validation of the use of double PO for ghosting analysis, a lens was analysed with 
standard PO, double PO, and the body of rotation method of moments (BoR-MoM) analyses. The frequency was reduced from $100 \mathrm{GHz}$ to $40 \mathrm{GHz}$ for the sake of the BoR-MoM. Cuts in the phi=45 degree plane are shown in Figure 4 for the copolar and cross-polar field. It is seen that the double PO (in which internal reflections in the lens are included) gives a significant improvement to the standard PO and is in good agreement with the (in principle exact) BoR-MoM. The results serve as a justification for using the double PO for ghosting analysis.

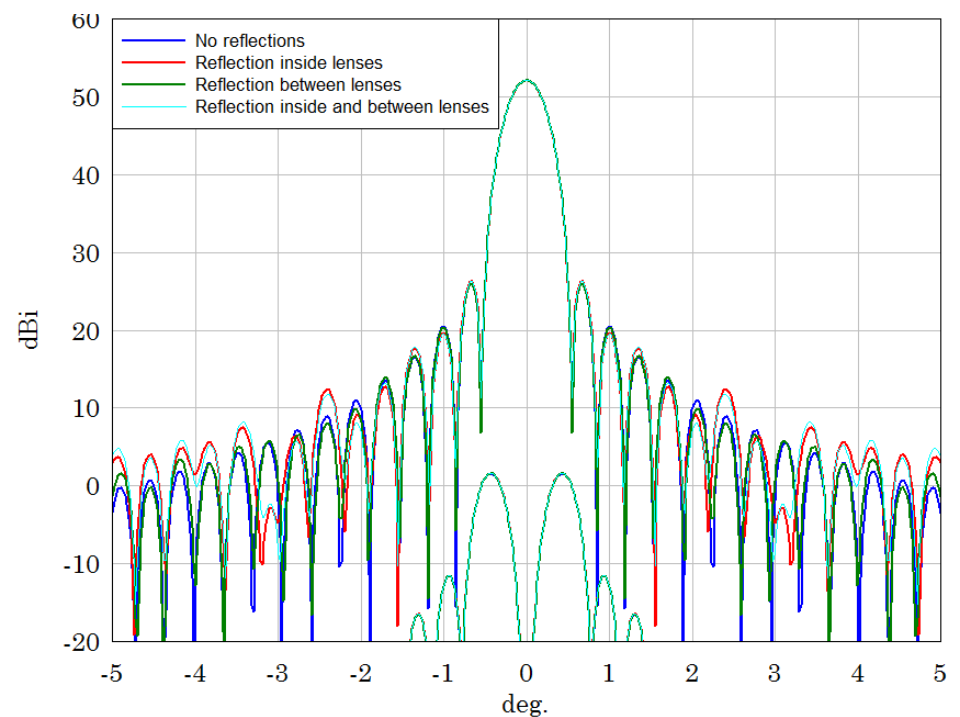

Figure 3. GRASP double PO analysis - effect of reflections between and inside lenses for baseline design.

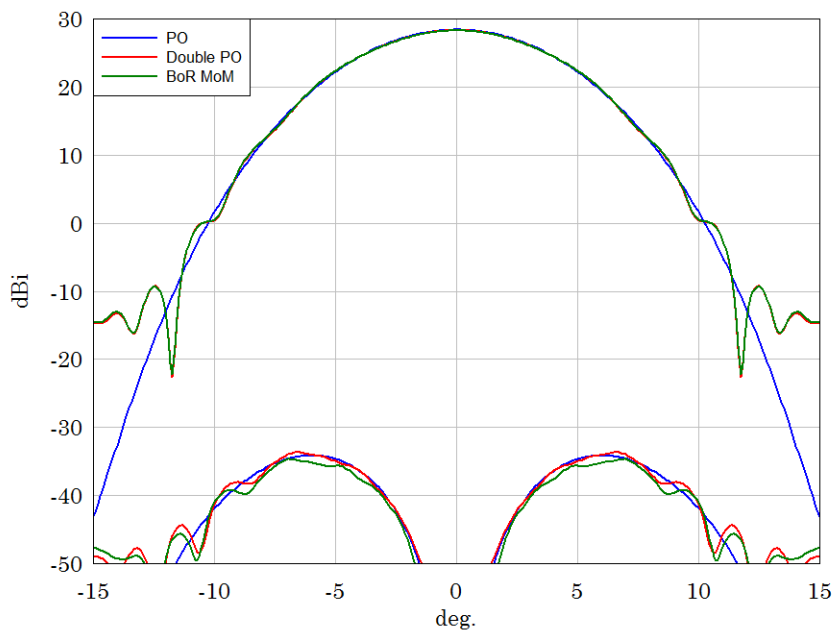

Figure 4.Comparison of standard PO, double PO and BoR-MoM for one of the baseline lenses, analysed at $40 \mathrm{GHz}$.

As expected, the level and integrated cross-polarization get worse as the edge of the field of view is reached (off-axis illumination of the telescope optics), however the levels are below $-40 \mathrm{~dB}$ even when multiple reflections are taken into account.

\subsection{Science impact of design solution}

The science performance of the full optical system was calculated using the methodology described in [7]. We analyzed five pixels in the focal plane: on axis; displaced by $50 \%$ of the focal plane radius with radial and tangential polarization; displaced by $50 \%$ of the focal plane radius with polarization at $\pm 45^{\circ}$ to radial; a marginal pixel with radial and tangential polarization; and a marginal pixel with polarization at $\pm 45^{\circ}$ to radial.

An example output is shown in Figure 5 and Figure 6. Figure 5 plots the $M_{Q T}$ and $M_{Q U}$ Müller fields, both of which vanish for an ideal system. The normalization is such that the integrated $M_{T T}$ field is unity. The axes of the far-field coordinate 
system are always aligned with the nominal polarization directions so that, for pixels with polarization rotated by $45^{\circ}$ to the radial, radial optical distortions appear at $45^{\circ}$. Figure 6 plots the spurious $B$-mode power induced by the optical imperfections and compares this with the goals and requirements established in [5].
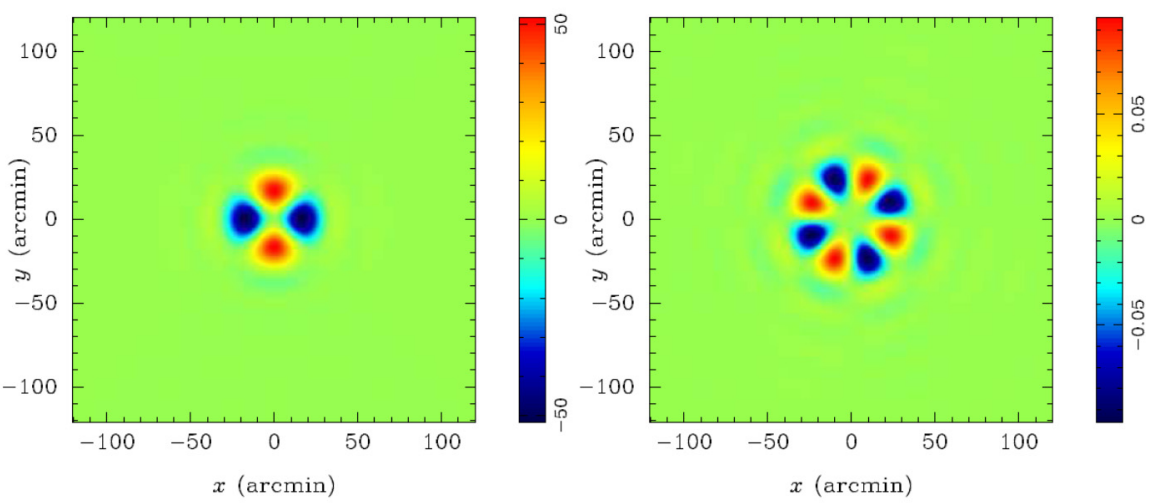

Figure 5. Müller fields $M_{Q T}(x)$ (left) and $M_{Q U}(x)$ (right) for an on-axis pixel in the 100-GHz baseline system with the mode-matched horn distribution as input. The integrated $\left|M_{Q T}\right|$ is $6.15 \times 10^{-3}$. The integrated $M_{Q U}$ is $4.32 \times 10^{-11}$ and the integrated $\left|M_{Q U}\right|$ is $1.81 \times 10^{-5}$

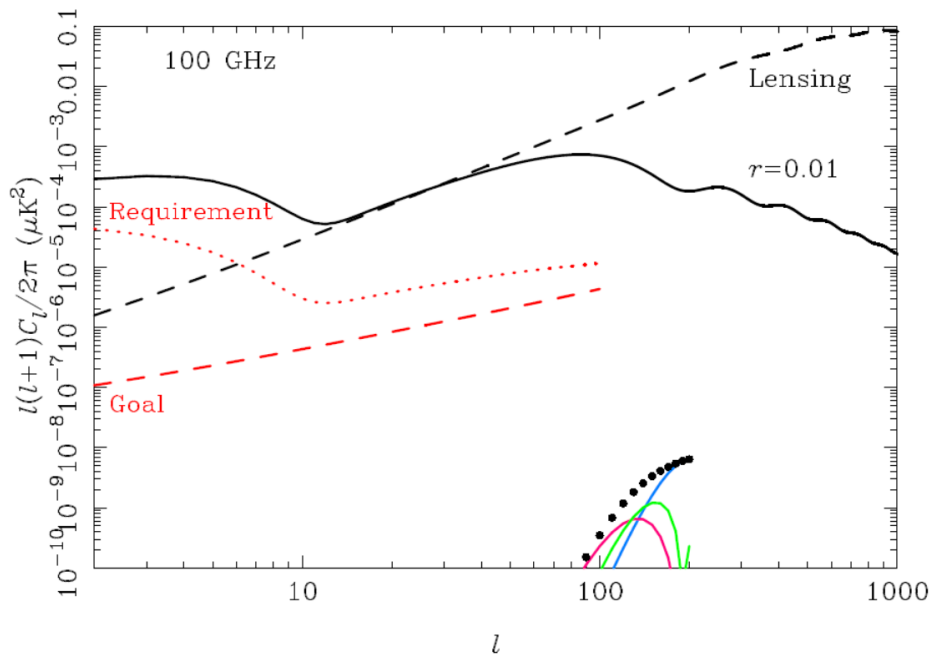

Figure 6. Spurious $B$-mode power (deconvolved with the nominal Gaussian beam) from the simulated beams illustrated in Fig. 3 . The requirements (red dotted) and goals (red dashed) on the level of spurious $B$-mode power are also plotted at this frequency (100 GHz). The total power is shown with points which is the sum of the power from $C_{l}^{T}$ (cyan), $C_{l}^{E}$ (magenta) and $C_{l}^{T E}$ (green). The spurious $B$ mode power is dominated here by $E \rightarrow B$ conversion for $l<100$, specifically $\nabla \nabla \nabla \nabla P \rightarrow P$ from the octupole of $M_{Q T}$. The $B$-mode power from weak lensing (black dashed) and primordial gravitational waves with $r=0.01$ (black solid) are also shown.

As expected from the results of the elliptical-beam fitting reported earlier, all pixels have excellent polarization purity and are within the goals for the system. Off-axis, the main source of spurious $B$-modes is from differential pointing (beam squint) between the two polarizations but this is well below 1 arcsec, even for the marginal pixels.

The differential ellipticity is always aligned with the polarization directions and $M_{Q T}$ is dominated by the ellipticity in the aperture fields rather than optical aberrations. The differential pointing does increase with distance from the axis but is within the goal specification. Finally, there is no evidence for significant common rotation between the two polarizations.

It should be noted that this excellent predicted performance is for the optimally aligned case. The performance degrades significantly for any misaligned optical elements, particularly in terms of differential pointing. The differential pointing would show up as an out-of-specification dipole in $M_{Q T}$ which would couple the gradient of the temperature to polarization. Such couplings are suppressed by the scan strategy to a degree, but not to a negligible level when components are out of alignment. Active modulation with e.g. a waveplate stepped such that any pixel on the sky is measured in the same 
instrument orientation, but with the polarization rotated by $90^{\circ}$ would null out the differential pointing effect. Alternatively, dipole-like modes can be projected from the multiple observations made of single sky pixels as part of the data analysis. The noise penalty in doing so does not seem significant for the fast-precessing scan strategy advocated for a future mission.

We recommend that a polarization modulator (e.g. HWP) be included in the baseline design to mitigate any issues with optical misalignment or other systematic effects.

\subsection{Instrument implementation}

For consideration of the maximum diameter for each telescope barrel, we allow a $20 \%$ margin on the diameter of the largest optical element in each system (L3). We also allow a dimension of $500 \mathrm{~mm}$ behind the focal plane for accommodation of the feedhorns, detectors and thermal architecture.

Table 3. Estimated telescope barrel volume envelopes.

\begin{tabular}{|l|c|c|}
\hline Telescope barrel dimensions & Bands 1, 2, 3 & Bands 4, 5 \\
\hline Diameter $(\mathbf{m})$ & 0.75 & 0.66 \\
\hline Length $(\mathbf{m})$ & 2.06 & 2.06 \\
\hline Volume $\left.\mathbf{( m}^{\mathbf{3}}\right)$ & 1.04 & 0.81 \\
\hline
\end{tabular}

We require five separate telescope barrels. These can be accommodated within a cylindrical volume envelope of diameter $2.1 \mathrm{~m}$, length $2.1 \mathrm{~m}$. These five parallel barrels would need to be tilted by $55^{\circ}$ with respect to the spacecraft spin axis in order to comply with the scan requirements. The optimal layout of telescope barrels requires further study. Assuming the use of ultra-high molecular weight polyethylene for the lens material, we derive the masses for the telescope barrels (lenses only, not supporting structure) indicated in Table 4.

Table 4. Mass estimates for the baseline design telescope barrels (lenses only)

\begin{tabular}{|l|c|}
\hline & Mass of lenses (kg) \\
\hline Bands 1, 2, 3 (each barrel) & 25 \\
\hline Bands 4, 5 (each barrel) & 17.5 \\
\hline Total (five barrels) & 110 \\
\hline
\end{tabular}

To derive requirements on component temperatures, we constructed an optical loading model to calculate the thermooptical load on the detectors from each component in the optical chain. Using a naiive instrument configuration we derive maximum component temperatures as indicated in Table 5.

Table 5 Maximum component temperatures permissible for the baseline detector numbers.

\begin{tabular}{|l|c|}
\hline Component & Temperature (K) \\
\hline Filter stack 1 & 14.0 \\
\hline HWP & 9.0 \\
\hline L1 & 5.0 \\
\hline L2 & 5.0 \\
\hline L3 & 5.0 \\
\hline Filter stack 2 & 0.2 \\
\hline
\end{tabular}

This model also indicates that a temperature fluctuation of the first optical element (sky-side, filter stack 1 ) of $0.3 \mathrm{~K}$ would present a change in power equivalent to a $10 \%$ change in the $\mathrm{NEP}_{\mathrm{CMB}}$. The requirements on temperature stability and the temporal scale of any fluctuation require further study.

\section{MATERIALS TESTING PROGRAMME}

At the beginning of this study, there were many questions as to the use of lenses in polarimetry experiments, such as:

- Good knowledge of refractive index as a function of temperature, and as a function of frequency

- Material uniformity

- Polarization effects - e.g. birefringence

- Uniformity and performance of impedance matching layer

- Thermal effects - coefficient of thermal expansion, conductivity, heat capacity 
The telescope design consists of ultra-high molecular weight polyethylene (UHMW-PE) lenses, with an impedance matching layer of porous PTFE on each surface. Material samples were tested optically at room temperature and at cryogenic temperatures. Measurements consisted of high precision spectroscopy of bi-planar samples in relevant spectral ranges, and the recovery of refractive index via the fitting of a Fabry-Perot model. Data analysis included modeling of system-related effects to maximize precision of the recovered parameters. This included correction for the thickness of the sample due to thermal expansion effects, correction for the converging beam of the Fourier Transform Spectrometer (FTS), temperature effects in the FTS position sensor etc. Full details can be found in [18].

A critical issue noted during the study was that there is significant variation in optical parameters, depending on the material batch, and on its treatment. For instance, there is a significant difference in optical parameters for material that has been drawn from the mill in rod form, compared to material that has been rolled as a slab. In particular, we found it critical to ensure that any material was annealed prior to manufacture, and samples of the same annealed material were tested optically (for refractive index), prior to the lens design being optimized. The annealing process also mitigated any potential shrinkage following the coating process (effectively another anneal cycle).

\section{LENS MANUFACTURING \& TESTING PROGRAMME}

Two different lens designs were manufactured. One type was optimized for the Cardiff tests (near-field re-focussing measurements and spectral efficiency tests) and was designated C-type. The second type was optimised for the Maynooth tests (VNA amplitude and phase tests - plane aperture field) and was designated M-type. Three copies of each lens type were built, and designated C1, C2, C3, M1, M2, and M3. This enabled monitoring of the repeatability of the manufacturing process, and provided redundancy to mitigate potential problems with the coating process. It also allowed some indication of the repeatability of the coating process. Lenses were manufactured by Ensinger Precision Engineering Ltd. All six lenses were visually inspected, and measured using the Cardiff surface measurement system (Mitutoyo coordinate measuring machine). All lenses underwent detailed 2-D grid scans, with an analytical expression fitted to each surface to recover surface parameters. This was done at all stages of lens processing. This was done to monitor any form changes induced by annealing the lenses, and by the coating process. We also designed and manufactured a third lens type as proof of manufacturing capability. This lens was a replica of the largest lens that would be required for the baseline telescope design presented in [6]. This lens has a diameter of $585 \mathrm{~mm}$. The agreement between the as-designed form and the fit to the mechanical measurements was excellent. At the perimeter of each surface, we found a maximum deviation from the ideal prescription of 86 microns for surface 1 , and 33 microns for surface 2.

For each of the C-type and M-type lenses, we carried out the following processing and test sequence:-

- $\quad$ Full enhanced grid CMM measurements of the surfaces - as-received

- Optical tests - as-machined

- Comparison with GRASP model (revised, fitted lens parameters)

- Anneal lens

- Full enhanced grid CMM measurements of the surfaces - post-annealing

- Optical tests - post-annealing

- Comparison with GRASP model (revised, fitted lens parameters)

- Coating

- $\quad$ Full enhanced grid CMM measurements of the surfaces - post-coating

- Optical tests - post-coating

- Comparison with GRASP model (revised, fitted lens parameters)

\section{LENS COATING PROCESS DEVELOPMENT}

Large UHMW-PE lenses have been successfully coated in the past by Hargrave \& Savini [19] using a vacuum pressing technique, but this process had issues with the application of differential pressure, and suffered from poor process control $\&$ repeatability. We have developed a new process for reliably coating lenses up to $600 \mathrm{~mm}$ diameter. We have designed and validated a new coating facility that allows one to coat both sides of a lens at the same time. This means that the lens never experiences differential pressure and minimizes the risk of distortion. Details of the process may be found in [18]. 

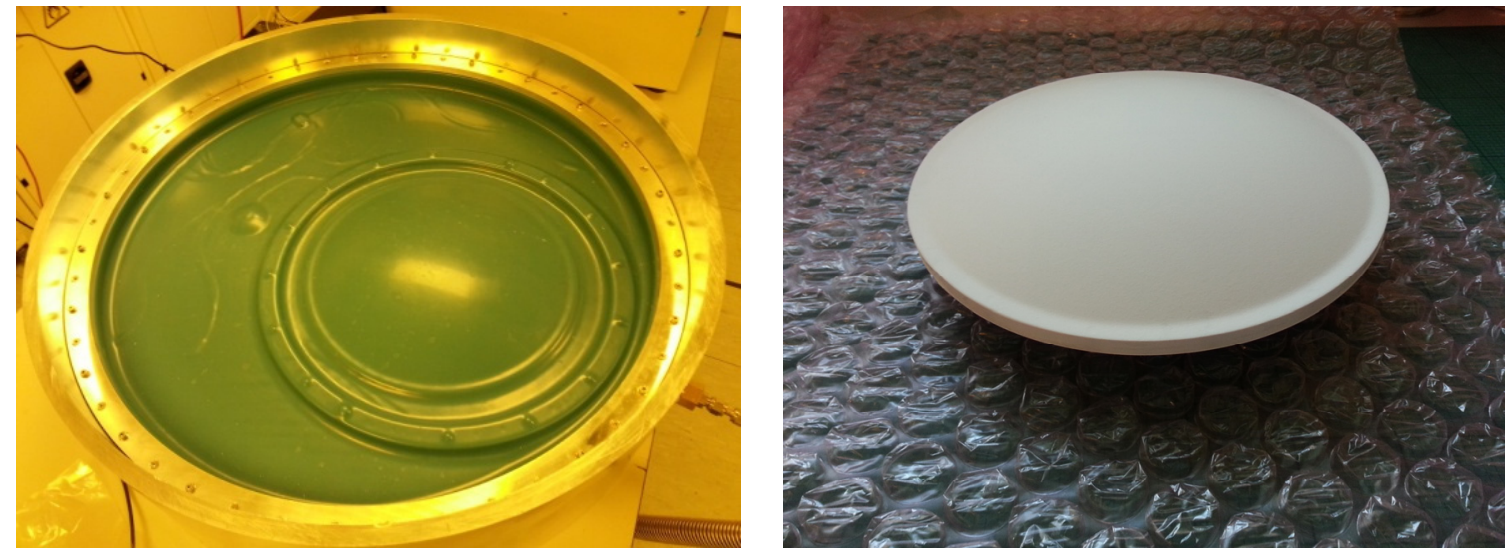

Figure 7. Left - image of coating rig. Right - example of a coated lens

\section{MODELLING TOOLS - COMPARISON OF PHYSICAL OPTICS AND BOR-MOM}

The software package GRASP was used for modeling, and analysis of experimental results. The two analysis methods, Physical Optics (PO) and Body of Revolution Method of Moments (BoR-MoM) were compared with respect to accuracy for the C-type and M-type lenses. The BoR-MoM is in principle exact, but time consuming for off-axis feeds. It is thus attractive to use the much faster PO when it is accurate enough. The PO method consists of applying the Fresnel refraction formulas on both surfaces of the lens in order to find equivalent currents that represent the field scattered by the lens. The PO can be further refined by means of the so-called Double PO which includes one additional set of reflections inside the lens. In contrast to this the BoR-MoM includes multiple interactions of all orders and the accuracy is only limited by the discretisation of the geometry. In Figure 8 the BoR-MoM is compared to PO and Double PO for the M-type lens with nominal geometry parameters and index of refraction. A similar analysis for the C-type lens was also performed.
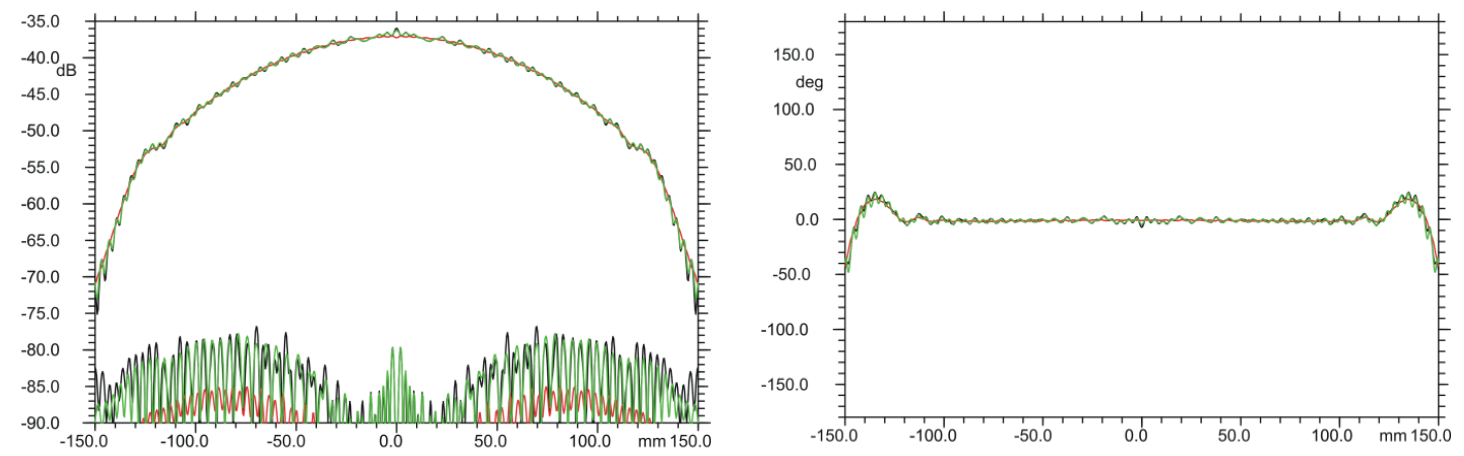

Figure 8. Left: Amplitude of co- and cross- components, $\phi=45^{\circ}$. Right: Phase of co-component of the E-field vector, $\phi=45^{\circ}$. Black: BoR-MoM. Red: PO. Green: Double PO. $100 \mathrm{GHz}$

It is seen that the BoR-MoM, PO and Double PO agrees very well for the co-polar amplitude, whereas the standard single PO is not sufficiently accurate for the cross-polarization. The internal reflection included in the Double PO is important. In general it is found that PO is sufficient for the co-polar component, but that BoR-MoM or Double PO is needed for accurate cross-polar prediction.

Now a coating layer is included in the model. The BoR-MoM is still solved without approximation, but in PO the simple Fresnel refraction must be replaced by refraction through a layered media. The coating is assumed to have the ideal parameters which is an index of refraction equal to the square root of that of the lens material and a thickness of $\lambda / 4$, where $\lambda$ is the wavelength in the coating material. The results are shown in Figure 9. 

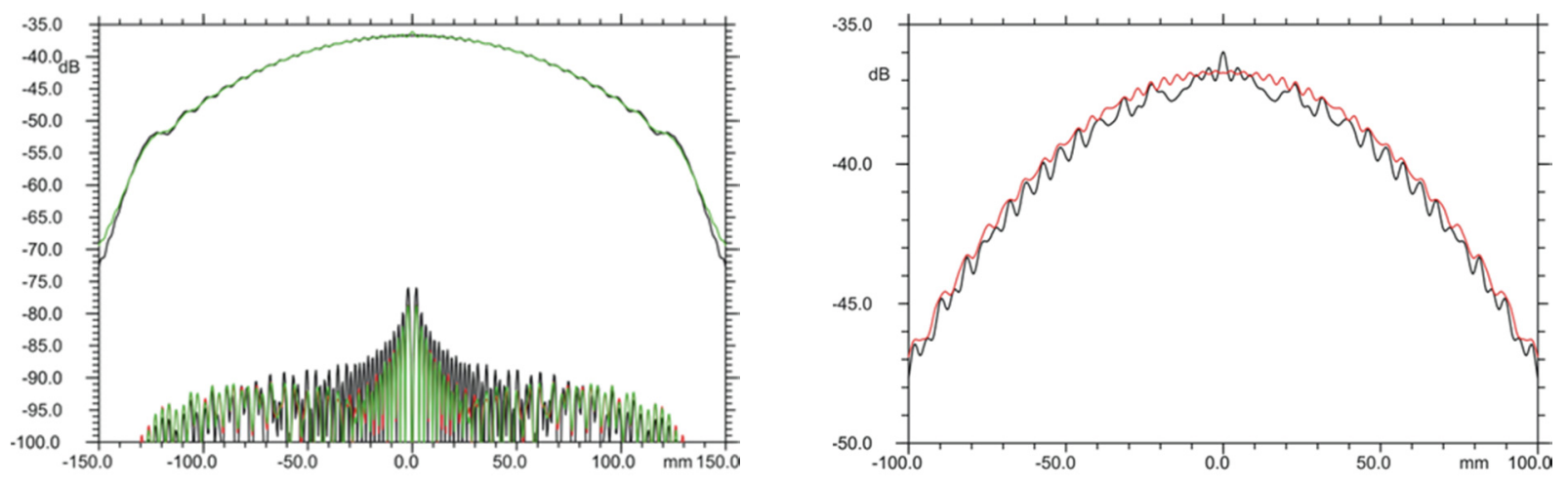

Figure 9. Left: Amplitude of co- and cross- components, $\phi=45^{\circ}$ for M-type lens with coating. Black: BoR-MoM. Red: PO. Green: Double PO. $100 \mathrm{GHz}$. Right: Amplitude of co-components, $\phi=45^{\circ}$ for M-type lens with and without coating. Black: BoR-MoM without coating. Red: BoR-MoM with coating. $100 \mathrm{GHz}$

All methods agree very well for the co-polar field, but that PO and Double PO underestimates the cross-polar close to the axis. Using BoR-MoM it is found that the central cross-polar lobes are mainly caused by edge diffractions and that the level of these lobes is sensitive to how the geometry of the coating is defined around the edge. It can be concluded that if the edge illumination is relatively high the general level of cross-polar field can be predicted well by PO, but the precise lobe structure must be computed by BoR-MoM with accurate modeling of the edge coating geometry and support structure. A close-up view of the co-polar field with and without coating is shown in the right-hand panel of Figure 9. It illustrates that coating results in higher field levels and smaller ripples due to less internal and external reflection.

\section{OPTICAL BEAM AND LENS PSF MEASUREMENTS}

The majority of optical tests were conducted using a Rhode \& Schwarz ZVA-24 vector network analyser, extended into the W-band (75-110 GHz) with a ZVA-ZV110 head. The system consisted of a feedhorn launcher and waveguide probe receiver, mounted on an optical bench. The probe could be scanned in three orthogonal directions, parallel and perpendicular to the launcher axis. Between the launcher and receiver was a rotary mounting system for the lens under test. Measurements and simulations were compared for all lenses, in all states (unannealed, annealed, coated) and configurations (on-axis, off-axis).

\subsection{M-type lenses}

Excellent agreement was found between measurements and simulation for the M-type lenses, apart from a small discrepancy in focal position for the unannealed, uncoated lens. Example output is shown for the coated M-type lens in Figure 10 to Figure 13.
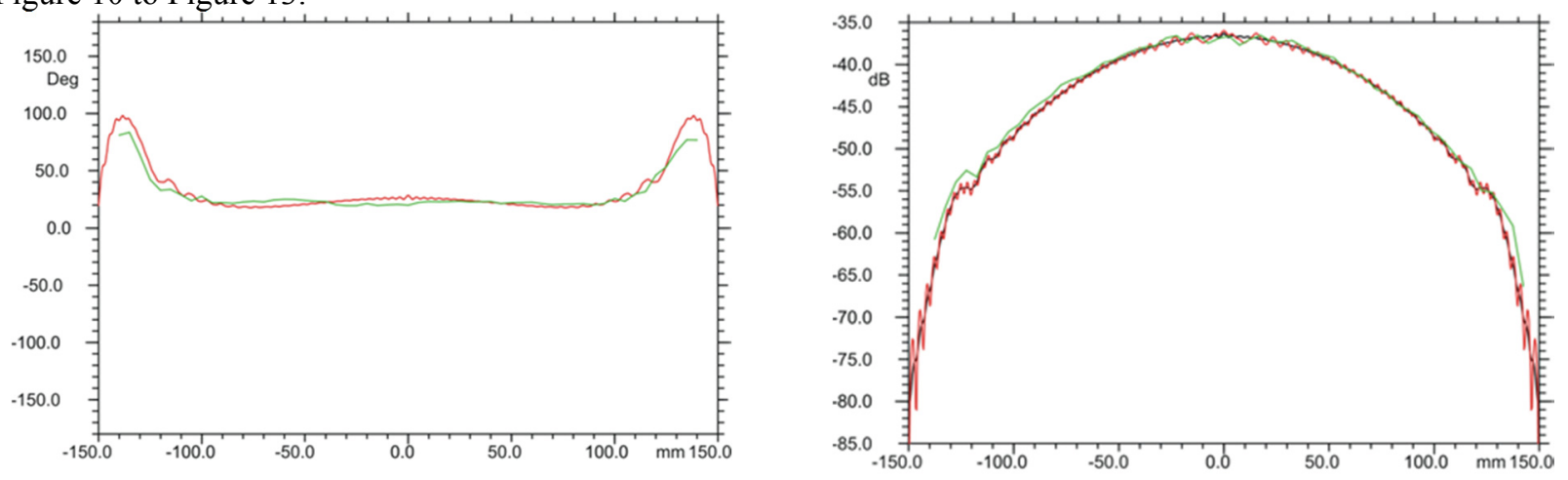

Figure 10. Left: Phase of co-component, $\phi=0^{\circ}, 100 \mathrm{GHz}$ Red: simulated, $\mathrm{z}=360 \mathrm{~mm}$. Green: measured, $\mathrm{z}=360.0 \mathrm{~mm}$. Right: Amplitude of co-component, $\phi=0^{\circ}, 100 \mathrm{GHz}$ Black: PO. Red: Double PO. Green: measured. 

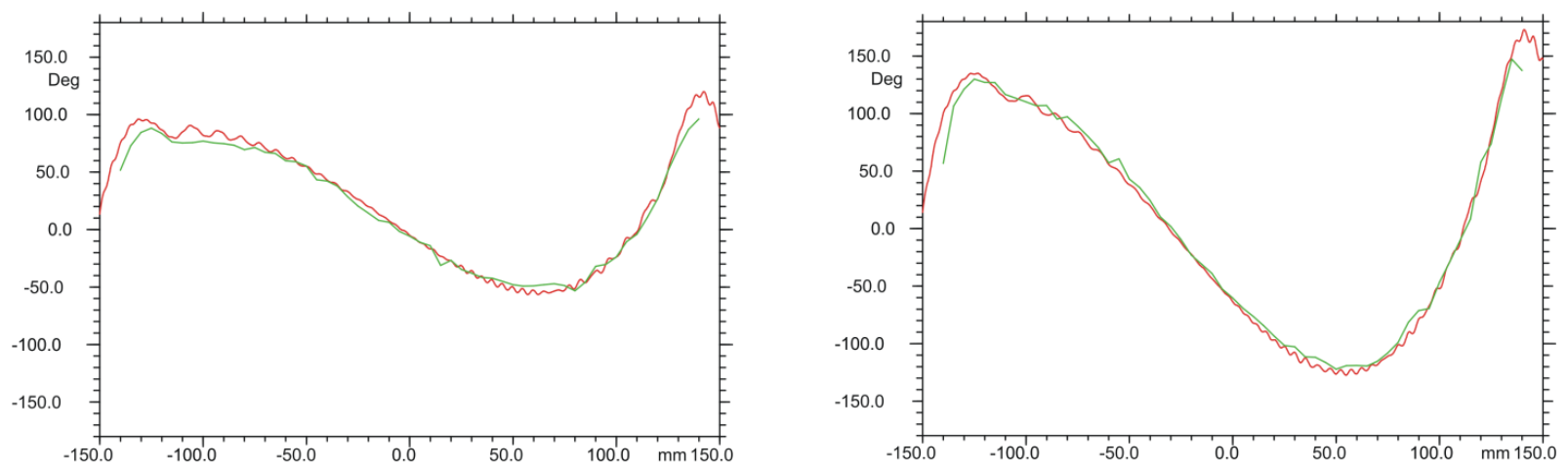

Figure 11. Phase of co-component, tilt $=10^{\circ}$ (left) $15^{\circ}$ (right), $75 \mathrm{GHz}$ Red: PO. Green: measured
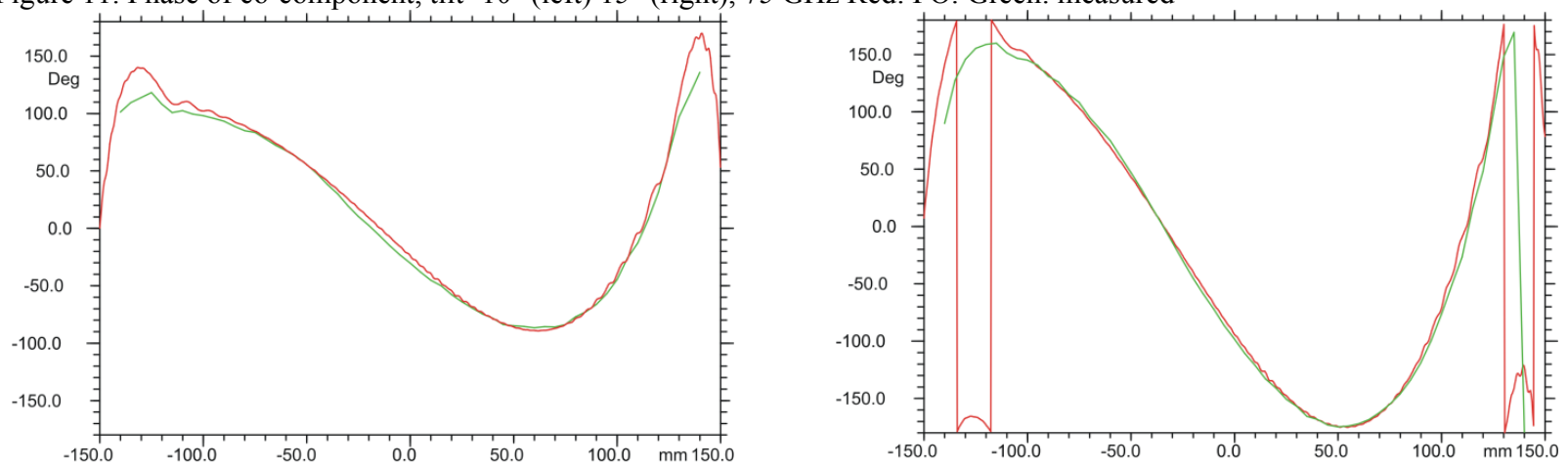

Figure 12. Phase of co-component, tilt $=10^{\circ}$ (left) $15^{\circ}$ (right), $100 \mathrm{GHz}$ Red: PO. Green: measured
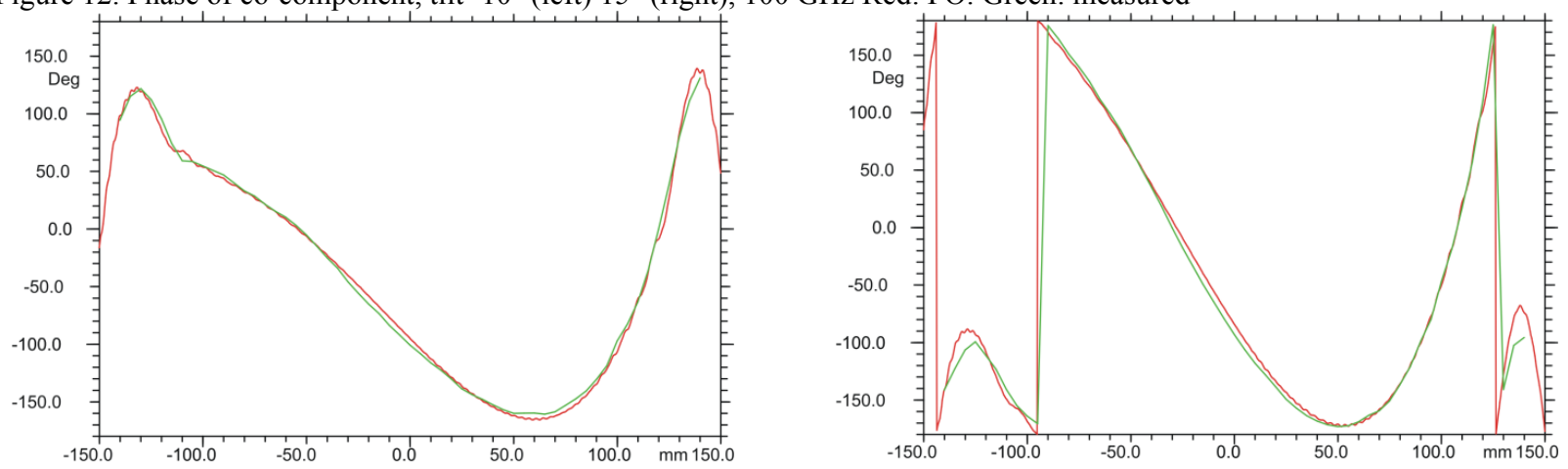

Figure 13. Phase of co-component, tilt $=10^{\circ}$ (left) $15^{\circ}$ (right), $110 \mathrm{GHz}$ Red: PO. Green: measured

\subsection{C-type lenses}

There was a larger discrepancy of order $20 \mathrm{~mm}$ noted between the experimentally-derived focal position, and that predicted by simulation for the C-type lenses. The discrepancy in the location of the focal point could be attributed to uncertainties in the value of the refractive index, the alignment accuracy and the accuracy of the measured lens surface parameters. But large errors in the above are considered unlikely, as there was excellent agreement for the M-type lenses. Further investigation of the measurement data revealed the presence of a clear standing wave effect. This is discussed further in [18].

Subsequent modeling was carried out for an output grid position offset $20 \mathrm{~mm}$ from the experimentally derived best focus. Some representative results for coated C-type lens configurations are shown in the following. The main lobe agrees reasonably well, but the sidelobe positions do not agree as well as expected. This is probably connected to the focal point discrepancy discussed above. Comparison with measurements for the optimum output grid position is shown in Figure 14. 


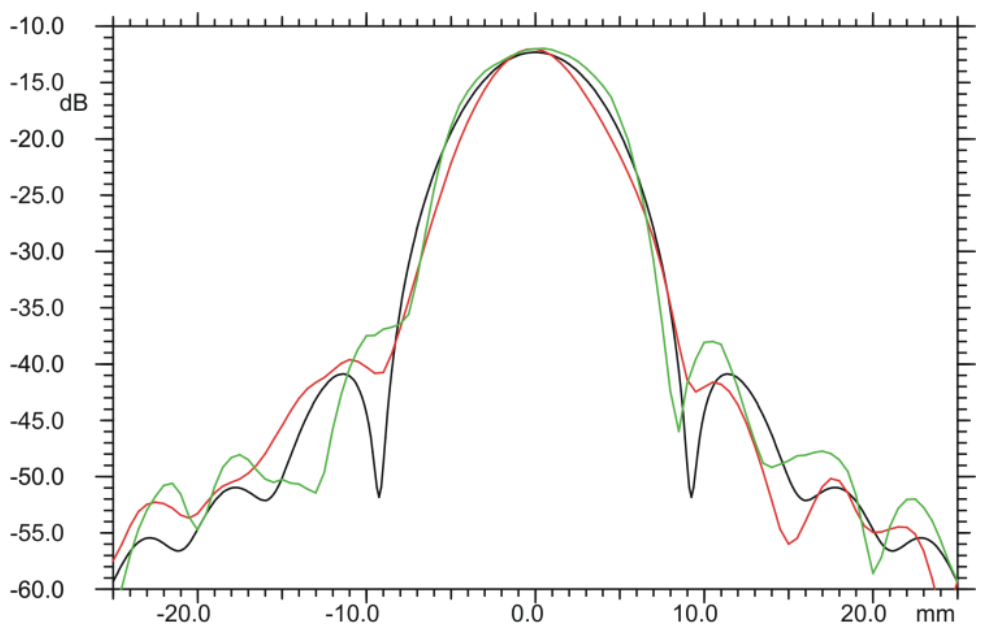

Figure 14. Amplitude of $\mathrm{C} 1$ co-component, $100 \mathrm{GHz}$

Black: simulation $\phi=0^{\circ}$. Red: measurement $\phi=0^{\circ}$. Green: measurement $\phi=90^{\circ}$.

Figure 14 shows that the focal point has been reached in the measurements and that the beam shape agrees reasonably well with the simulation. The measured sidelobes are higher and asymmetric, and the $\phi=0^{\circ}$ and $\phi=90^{\circ}$ cuts differ, whereas they are nearly identical in the simulations.
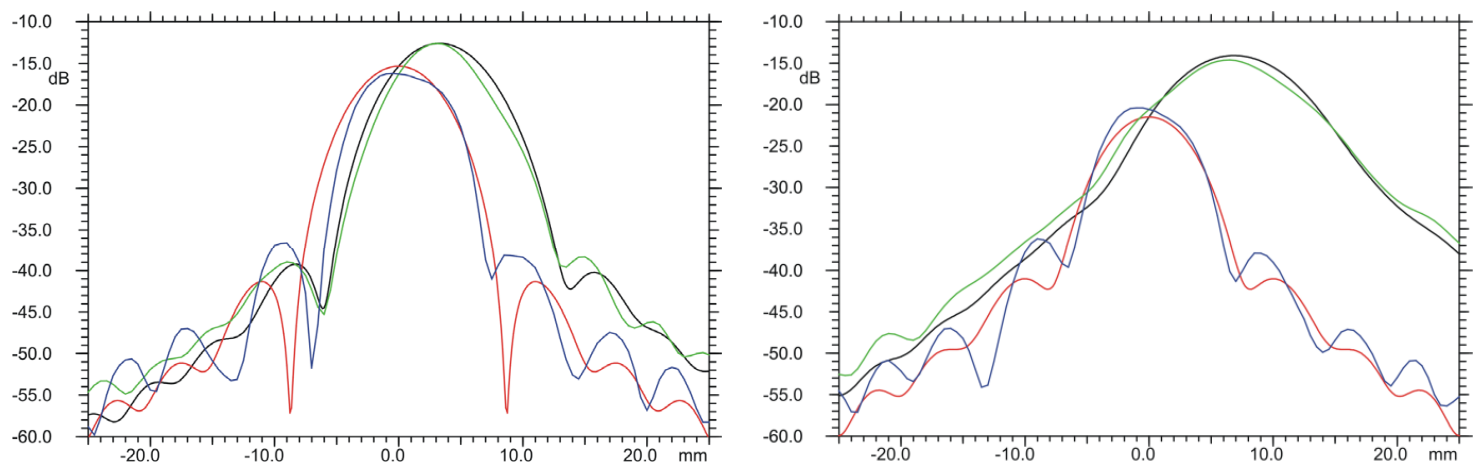

Figure 15. Amplitude of co-component, tilt $=5^{\circ}$ (left) $10^{\circ}$ (right), $100 \mathrm{GHz}$

Black: PO, $\phi=0^{\circ}$. Red: PO, $\phi=90^{\circ}$. Green: measured, $\phi=0^{\circ}$. Blue: measured, $\phi=90^{\circ}$.

\section{SUMMARY AND KEY FINDINGS}

\subsection{Material characterization}

We have developed robust and repeatable measurement and analysis procedures for the recovery of refractive index and birefringence values to very high levels of precision, at around the $1 \times 10^{-4}$ level, depending on the sample parameters and frequency range.

\subsection{Material heritage and processing}

Best available knowledge of the material heritage is very important, and the generic material certificates of conformance supplied by machining firms are not sufficient. Knowledge of the processing of the material in the production mill is very important, such as the extrusion/rolling direction, heat treatment etc.

The substrate material must be annealed prior to lens manufacture. Otherwise the coating process will anneal the manufactured lens and change the surface form.

We have developed and tested an annealing cycle suitable for lenses made from UHMW-PE, after which the lenses remain stable with subsequent heating cycles. 


\subsection{Lens manufacture and mechanical characterization}

Lenses must be manufactured from annealed material. The material from which lenses are to be machined must be fully processed and characterized BEFORE the lenses are designed and manufactured.

We have confirmed that lenses can be manufactured to the dimensions required for the baseline telescope design presented in [6] with a high degree of conformance to the design intent.

Additionally, we have developed a robust process for mechanical characterization and analysis of lenses in uncoated and coated forms.

\subsection{Lens coating}

We have developed and validated a reliable and repeatable process and thermal model for coating large lenses up to 600 mm diameter.

However, it has recently become difficult to source coating material (porous-PTFE) in sheets wider than $330 \mathrm{~mm}$. For large lenses, we may have to consider refining and further validating this process by using multiple sheets to cover the required area.

\subsection{Lens optical characterization and optical model validation}

We have successfully tested two types of lens in various stages of processing, recovering both phase and amplitude information. The experimental results have been extremely well modelled by the newly-developed software module (QUAST) for GRASP, using both "body of rotation method of moments" (BOR-MOM) and physical optics (PO) analyses. This modelling required good knowledge of the mechanical form and refractive index of all lenses at all stages of processing, and it is seen as a good validation of both the software, and of the experimental programme.

\section{ACKNOWLEDGEMENTS}

This work was funded by the European Space Agency under contract number 4000102522/10/NL/AF.

\section{REFERENCES}

1. Ade, P., et al., BICEP2 I: Detection Of B-mode Polarization at Degree Angular Scales. arXiv preprint arXiv:1403.3985, 2014.

2. Hanson, D., et al., Detection of B-mode polarization in the Cosmic Microwave Background with data from the South Pole Telescope. Physical Review Letters, 2013. 111(14): p. 141301.

3. Armitage-Caplan, C., et al., COrE (Cosmic Origins Explorer) A White Paper. arXiv preprint arXiv:1102.2181, 2011.

4. Bernardis, P., et al., B-Pol: detecting primordial gravitational waves generated during inflation. Experimental Astronomy, 2009. 23(1): p. 5-16.

5. Hargrave, P., A.D. Challinor, and T. Peacocke, Review and consolidation of telescope instrument requirements (TN1), in Modular Wide Field of View RF Configurations - ESTEC contract number 4000102522/10/NL/AF2011, Cardiff University.

6. Hargrave, P., et al., Telescope baseline design report (TN3), in Modular Wide Field of View RF Configurations - ESTEC contract number 4000102522/10/NL/AF2012, Cardiff University.

7. Hargrave, P., et al., Telescope configuration trade-off report (TN2), in Modular Wide Field of View RF Configurations - ESTEC contract number 4000102522/10/NL/AF2011, Cardiff University.

8. Hargrave, P. and G. Savini, Critical components and technologies identification (TN4), in Modular Wide Field of View RF Configurations - ESTEC contract number 4000102522/10/NL/AF2012, Cardiff University.

9. Hargrave, P. and G. Savini, Critical Breadboards Definition and Analysis (TN5), in Modular Wide Field of View RF Configurations - ESTEC contract number 4000102522/10/NL/AF2012, Cardiff University.

10. Ade, P.A.R., et al. A review of metal mesh filters. 2006. 
11. Ade, P., R. Wylde, and J. Zhang. Ultra-Gaussian Horns for ClOVER-a B-Mode CMB Experiment. in Twentieth International Symposium on Space Terahertz Technology, edited by: E. Bryerton, A. Kerr, and A.

Lichtenberger, Charlottesville. 2009.

12. Lewis, A. and A. Challinor, Weak gravitational lensing of the CMB. Physics Reports, 2006. 429(1): p. 1-65.

13. Smith, K.M., et al., CMBPol mission concept study: gravitational lensing. arXiv preprint arXiv:0811.3916, 2008.

14. Bock, J., et al., Study of the Experimental Probe of Inflationary Cosmology (EPIC)-Intemediate Mission for NASA's Einstein Inflation Probe. arXiv preprint arXiv:0906.1188, 2009.

15. Bock, J., et al., The Experimental Probe of Inflationary Cosmology (EPIC): A Mission Concept Study for NASA's Einstein Inflation Probe. arXiv preprint arXiv:0805.4207, 2008.

16. Babich, D. and M. Zaldarriaga, Primordial bispectrum information from CMB polarization. Physical Review D, 2004. 70(8): p. 083005.

17. Candotti, M., et al., Mm and sub-mm Complete Refractive Telescope Electromagnetic Design for High Purity Electromagnetic Wave Polarization Detection, in ESA Workshop on large deployable antennas2012, ESA: ESTEC.

18. Hargrave, P., et al., Critical breadboards manufacture and test report (TN6), in Modular Wide Field of View RF Configurations - ESTEC contract number 4000102522/10/NL/AF2013.

19. Hargrave, P.C. and G. Savini, Anti-reflection coating of large-format lenses for sub-mm applications, in Society of Photo-Optical Instrumentation Engineers (SPIE) Conference Series2010. p. 20. 